\title{
Comparación de las variaciones de la composición corporal entre judokas y luchadores hidratados Vs deshidratados

\author{
Comparison of body composition variations between hydrated and dehydrated judokas and wrestler \\ *Bibiana Calvo Rico, **Luis Fernandes Monteiro, Susana Aznar Laín, José Manuel García García \\ *Universidad de Castilla-La Mancha (España), **Universidad Lusófona (Portugal)
}

\begin{abstract}
Resumen. El propósito de este estudio fue conocer cómo se modifican los parámetros de composición corporal en deportes como judo y lucha, ambos deportes divididos por categorías de peso en los cuales, de manera habitual se producen desórdenes alimentarios y continuas deshidrataciones para la búsqueda del peso de competición. Igualmente se trató de conocer si existían diferencias entre hombres y mujeres en esta práctica y diferencias entre ambos deportes. Para ello, se utilizó el sistema Inbody Biospace 230 (CA,USA) a una muestra de élite nacional de judo y lucha del equipo español y portugués de 64 deportistas $(\mathrm{n}=64)$. El análisis estadístico se ejecutó mediante las pruebas: Kolmogorov-Smirnov y t de students para analizarlas diferencias de medias. Los resultados y conclusiones del estudio demostraron entre otros, que el número de atletas que se encontraban deshidratados en el momento pre-competitivo es mayor que los que están en valores óptimos de hidratación, sometiendo al organismo a constantes esfuerzos y cambios, pudiendo ser este un efecto perjudicial a corto y largo plazo en el rendimiento para el atleta, tanto en el entrenamientos como para la propia competición.
\end{abstract}

Palabras Clave: Deshidratación, Inbody Composición Corporal, Judo, Lucha y peso de competición.

Abstract. The aim of this study was to determine how body composition parameters are modified in judo and wrestling. Both disciplines are organized by weight categories, this regularly leading to eating disorders and continuous dehydration in order to reach the needed competition weight. Also, this paper aims to understand whether there exist differences in this practice by gender and by sports discipline. A Biospace Inbody 230 system (CA, USA) was employed on a sample of 64 national elite judo and wrestling athletes from Spain and Portugal. Statistical analysis was performed using Kolmogorov-Smirnov and student $t$ tests, which analyze mean differences. The findings and conclusions of the study showed a higher number of dehydrated athletes than those in optimal values of hydration during the pre-competitive period. This condition pushes athletes' body to constant efforts and changes, possibly determining a detrimental effect on their short- and long-term performance, both in training and competitions.

Key Words: Dehydration, Inbody Body Composition, Judo, Wrestler, competition weight.

\section{Introducción}

Cuando nos referimos a deportes en los que las categorías de peso son fundamentales para el desarrollo de la competición, debemos tener en cuenta de qué manera afecta la nutrición elegida al rendimiento. En concreto, el tipo de dieta elegida, la restricción alimenticia, la disminución en la ingesta de líquidos, etc.., son una parte fundamental de la nutrición y si se realizan de forma incorrecta, pueden llevar a diferentes trastornos nutricionales e incluso a estados de deshidratación que afecten al rendimiento del deportista.

Los niveles óptimos nutricionales han sido objeto de estudio por toda la sociedad durante el paso de los años ya fuera en el ámbito sociológico como deportivo, en población infantily adolescente, como adulta y/o en la vejez.

Thield et al. (1993), observaron cómo aproximadamente un 52\% de remeros y luchadores recurrían frecuentemente a los vómitos para perder peso.

Franseen (1997), en sus investigaciones sobre los desórdenes alimenticios en atletas femeninos, analizaba como aquellos delimitados por categorías de peso utilizaban más el uso de dietas, prácticas de pérdidas de peso patológicas y deseo de adelgazar que en otros deportes donde no existía la diferencia de categorías por peso.

En 1999, Rodríguez et al. realizaron un estudio con población adolescente donde trataban de desarrollar una relación directa con las prácticas y conductas alimentarias alteradas en deportes individuales. Fue entonces, cuando apareció el concepto de «Anorexia por Actividad» donde el sujeto busca en la actividad física la pérdida de peso de manera compulsiva. Aquellos sujetos que practicaban deportes individuales, forzaban su organismo más allá de los límites biológicos, con el fin de ajustarse a unos cánones específicos de su deporte o de belleza. En dicho estudio, un $46 \%$ manifestó haberse provocado el vómito alguna vez con fines «dietéticos», entre 1 y 3 veces al día y un $26 \%$ había utilizado alguna vez algún tipo de laxantes con intención de perder peso, ocurriendo este dato 2,56 veces más en sujetos practicantes de deportes

Fecha recepción: 08-10-16. Fecha de aceptación: 16-02-17

Becha recepción.

Bibiana.Calvo@uclm.es individuales, los cuales además de períodos purgativos podían pasar tiempos de ayuno, que en los practicantes de deportes colectivos.

Kiningham \& Gorenflo (2001) realizaron un estudio para analizar que ocurría con la población más joven. 2532 luchadores en la escuela secundaria fueron encuestados mostrando que un $72 \%$ estaban involucrados en al menos un método de pérdida de peso potencialmente dañino a la semana de la temporada de lucha, un $52 \%$, utilizaba al menos dos métodos y el 12\% restante, utilizaba al menos 5 métodos cada semana. El uso semanal de laxantes, vómitos, pastillas de dieta o diuréticos se reveló únicamente en un $2 \%$ de los atletas. Los métodos de ayuno y purga, en busca de la deshidratación fueron los predominantes a lo hora de la búsqueda del peso de categoría óptimo.

Engels, Johnson et al. (2003) encontraron que los luchadores, mostraban una especial predisposición y tendencia a adelgazar, a la restricción alimentaria y a las conductas de purga, comparándolos con atletas de otros deportes.

En 2004, Juzwiak \& Ancona, se plantearon dar un paso más y evaluar los conocimientos sobre nutrición que poseían los entrenadores de varios deportes centrados en adolescentes. Buscaban como objetivo, describir las prácticas de alimentación recomendadas por los preparadores a sus deportistas para posteriormente, poder realizar las posibles recomendaciones dietéticas necesarias. A través de un cuestionario, se obtuvieron los conocimientos de los entrenadores y los resultados mostraron que, sí existían recomendaciones dietéticas generales durante los periodos de entrenamientos, pero sin especificar, estrategias: antes, durante y después de dichos periodos.

Sundgot-Borgen \& Torstveit (2004), investigaron sobre trastornos alimenticios y deporte en población deportista de élite en Noruega. Su estudio se dirigió hacia problemas como la anorexia nerviosa ${ }^{1}$, la bulimia nerviosa ${ }^{2}$ o la anorexia atlética ${ }^{3}$. Concluyeron que el $42 \%$ de los deportes de carácter estético tenían algún tipo de estos problemas superando a los deportistas de trabajo de resistencia. En dichos deportes, la característica fundamental que primaba el los deportistas era la extrema delgadez, siendo la población femenina la más afectada por dichos trastornos alimenticios.

Dossil (2004), recoge en su libro sobre psicología relacionado con la actividad física y el deporte, una breve clasificación de los deportes que están considerados de mayor riesgo por su problemática en las conduc- 
tas alimentarias, donde encontramos:

Deportes por Categorías: hace referencia a aquellos donde el peso delimita y condiciona la participación, por lo que el deportista trata de disminuir su peso para poder competir utilizando dietas restrictivas o algún tipo de purga para llegar al peso de competición. Podemos ejemplificar este tipo de deportes con: el judo, la lucha o el boxeo.

Deportes de Estética: claramente relacionados con aquellos deportes donde la figura y el aspecto físico del deportista puede influir en la valoración o nota recibida por parte de los jueces y árbitros. La estética que prima hace referencia a cuerpos fibrosos, caderas estrechas, poco pecho y piernas largas. Los más característicos son: gimnasia rítmica o el patinaje artístico.

Deportes de Gimnasio: donde puede aparecer la vigorexia ${ }^{4}$ normalmente en actividad física no reglada y normalmente no controlada por un especialista. Por ejemplo: el fitness, el culturismo, el aeróbic.. .

Deportes de Resistencia: aquellos donde el deportista, por las características del deporte de carácter eminentemente aeróbico, se puede llegar a beneficiar y bajar peso para mejorar con ello su rendimiento. Podemos observar entre ellos: el atletismo, la natación o el ciclismo.

Isabel Díaz Ceballos (2005), realiza una propuesta de un programa de prevención de trastornos alimentarios dirigida a los entrenadores, incidiendo en aquellos deportes que de manera implícita, pueden tener más riesgo, y a desarrollar un posible trastorno en la conducta alimenticia.

Estudios realizados en población adolescente, realizados por Boisseau et al. (2005), demuestran como los desequilibrios nutricionales en judokas en edades comprendidas entre los 15 y los 17 años, pueden modificar los estados de crecimiento y maduración en este grupo de edad. Cambios en la ingesta, tanto de comidas como de fluidos, en un período comprendido entre 3 y 1 semana previa a la competición, afectaban significativamente, provocando una deshidratación negativa en el adolescente, como consecuencia de la necesidad de adoptar un peso determinado acorde con la categoría específica del competidor.

Márquez (2008) relacionó dichos trastornos con manifestaciones de baja autoestima, distorsión de la imagen corporal, ineficiencia, perfeccionismo y pérdida de control, todo ello realizado con un mecanismo compensatorio ejercido a través de la manipulación de la comida. Muchos deportistas objeto del estudio, evitaban la supervisión por parte del entrenador o equipo médico, por miedo a que fueran descubiertos en sus métodos mecanismos inapropiados de adelgazamiento.

Müller (2009), realizó una clasificación de los deportes en función de los problemas asociados al peso y composición corporal, relacionados con los trastornos alimenticios. En ella, podemos encontrarnos:

Deportes en los que el peso limita el rendimiento debido a la mecánica del mismo, como: los saltos de esquí, la escalada deportiva, etc. . .

Deportes en los que las categorías de peso pueden ser perjudiciales a corto plazo, asociados a la deshidratación extrema, observando cómo los atletas esperan una ventaja cuando se clasifican en una categoría de peso inferior. Este grupo incluye: la lucha, el judo, el boxeo, taekwondo, etc. .

Deportes estéticos, en el que los deportistas esperan que sus puntuaciones sean mayores gracias a su reducción del peso corporal, y su percepción estética del cuerpo cumpla unos cánones ideales, como por ejemplo: gimnasiarítmica, patinaje artístico, natación sincronizada, etc. .

Cuando los trastornos alimenticios hacen su aparición por exigencias de la competición o del máximo rendimiento del deportista, se ve afectada la ingesta de vitaminas y minerales en nuestro cuerpo. Todos estos micronutrientes, son esenciales para la producción de energía que posteriormente el atleta necesita de manera obligada, no sólo para rendir en los entrenamientos propuestos por sus entrenadores, sino, a posteriori, en la propia competición.

No podemos olvidar, que tanto las vitaminas como los minerales, benefician y colaboran en la restauración de los tejidos musculares durante la recuperación del ejercicio, pero sobre todo, juegan un papel esencial en el restablecimiento de las lesiones deportivas, aspecto fundamental cuando de la alta competición se trata.

Un estudio realizado por Rodriguez \& DiMarco (2009), muestra como tanto el calcio, la vitamina D, B el hierro, Zinc como el Magnesio, resultan de gran interés para las dietas de los deportistas de alto nivel, sobre todo, para aquellos que corren un riesgo mayor al restringir el consumo de micronutrientes en su dieta debido a una deshidratación precompetitiva por búsqueda del peso de competición.

En 2010, Matías et al. describen: como la deficiencia del magnesio afecta en gran medida el rendimiento muscular, en concreto a la fuerza de la musculatura prensora de la mano, de vital importancia para un deporte como el judo, donde el kumikata ${ }^{5}$ es esencial para el desarrollo del combate.

Por otro lado, existen estudios que no encontraron sujetos con pérdida de peso, incluso estando moderadamente por encima de su peso, o categoría de competición, aun realizando modificaciones en su dieta, sin control por parte de un profesional (Úbeda et al. 2010)).

De Barros et al. (2010), buscaron un posible impacto entre los deportistas (judokas) con altos niveles de deshidratación y su relación con el hambre y la tasa de apetito. Midiendo los valores de hambre del deportista antes y después los entrenamientos demostrando que son mayores y más significativos, los niveles de sensación de hambre en sujetos deshidratados tras el entrenamiento y que éstos mostraban altos valores en el test de necesidad de frutas. Por ello, los cambios en el estado de hidratación de un atleta, pueden estar asociados a la percepción subjetiva de hambre y sed de ciertos alimentos.

Ööpik et al. en 2013 preocupados por los graves estados de deshidratación de los luchadores greco-romanos, analizaron en una situación real de competición a través de pruebas de orina, los niveles de hidratación de los deportistas participantes en el Campeonato de Estonia 2009. Los resultados mostraron como el $60 \%$ de la muestra redujo su masa corporal a través de la deshidratación previo a la competición, siendo este hecho un acto frecuente en esta disciplina deportiva.

\section{Objetivos}

a) Conocer cómo afectan al peso corporal los métodos de pérdida de peso realizados por los atletas durante la etapa previa a la competición; b) Conocer cómo afectan al porcentaje de grasa corporal los métodos de pérdida de peso realizados por los atletas durante la etapa previa a la competición; c) Analizar si existen diferencias entre hombres y mujeres en ambos parámetros para ver la importancia de los efectos nutricionales de la pérdida de peso corporal.

\section{Métodos}

Nos encontramos frente a un estudio descriptivo poblacional siendo una investigación aprobada por el ComitéÉtico del Vicerrectorado de Investigación y Política Científica de la UCLM en concordancia con la última versión de la declaración de Helsinki.

\section{Muestra}

La muestra se realizó con los equipos nacionales de judo y lucha de España y Portugal. Se eligieron tres concentraciones nacionales, una que se realizó en Portugal, concretamente en el Centro deAlto Rendimiento de Lisboa y dos en España, en los Centros de Alto Rendimiento de Madrid y de Las Aguilas (Murcia)

Se registraron 64 deportistas ( $\mathrm{n}=64$ ), de los cuales 38 eran luchadores $(n=38)$ todos ellos deportistas de alto nivel, los cuales formaban parte de toda la Selección Española de Lucha a nivel tanto Junior como Senior. El resto de sujetos eran judokas (n=26), de la Selección Portuguesa de Judo coincidiendo en el nivel con los luchadores teniendo una edad media de 21,76 $\pm 5,15$ años. El peso medio de los sujetos era $67,10 \pm 6,88$, incluidos hombres y mujeres en ambas modalidades deportivas.

\section{Material}

Para la evaluación de los diversos parámetros de composición corporal se utilizó el sistema Inbody Biospace 230 (CA,USA).

La evaluación de los participantes se realizó en el horario previo a las sesiones de entrenamiento de la tarde, coincidiendo con la franja 
horaria del pesaje de las competiciones de luchadores y judokas en los campeonatos.

Se acercaron las mediciones a la realidad pre-competitiva, y se siguieron en todos los casos las recomendaciones vertidas por distintos investigadores. De esta forma, los luchadores realizaron la medición con el maillot de lucha y descalzos, tal y como han de hacerlo en el pesaje oficial en competiciones nacionales e internacionales, sin embargo los judokas lo hicieron todos en ropa interior, aunque en los pesajes oficiales podrían subir a la báscula desnudos.

Se les evaluó sin haber realizado ninguna actividad física, toma de alimentos o líquidos 2 horas antes de la medición tal y como recomienda Martín Moreno et al. (2001). Así mismo, y tal como orienta Marrodán et al. (2007) se les dijo a los deportistas que antes de realizar la medición, orinaran y defecaran para estar sin residuos. En el momento de la evaluación los deportistas se situaban de pie, apoyando el talón y el metatarso sobre los electrodos de la base del Inbody 230 para cada pie. Con las manos, agarraban los electrodos situando los brazos separados del tronco. Los electrodos se limpiaban y secaban antes y después de cada una de las mediciones, así como pies y manos de los sujetos. (Clemente \& González Ravé, 2010).

La toma de datos se realizó durante el mes de Marzo del 2014. Todos los participantes firmaron su consentimiento previo a la toma de datos.

\section{Analísis estadistico}

Se comprobó la normalidad de la muestra para poder utilizar los test paramétricos mediante la prueba de Kolmogorov-Smirnov. Para el análisis de los resultados se utilizó el paquete estadístico SPSS v 20 y se utilizó estadística de carácter descriptivo, utilizando la t de students para analizarlas diferencias de medias.

\section{Resultados}

Los resultados en la etapa cercana a la competición mostraron como el porcentaje de grasa corporal presentaba diferencias significativas tanto para los atletas que se encontraban en niveles de hidratación $(p=0.022)$ como para los deportes de combate $(p=0.078)$ pero sin embargo no se encontraron diferencias significativas en lo que se refiere

\begin{tabular}{|c|c|c|c|c|c|c|c|}
\hline \\
\hline & & $\mathrm{N}$ & Media & $\begin{array}{l}\text { Desviación } \\
\text { Típica }\end{array}$ & $\begin{array}{l}\text { Error típico } \\
\text { de la Media }\end{array}$ & $\mathrm{t}$ & $p$ \\
\hline \multicolumn{8}{|c|}{ Composición Corporal por Niveles de Hidratación } \\
\hline \multirow{2}{*}{ Peso Precomp } & Hidratados & 17 & 65.69 & 13.91 & 3.59 & $1.38 \quad 60$ & \\
\hline & Deshidrat. & 47 & 71.79 & 15.00 & 2.18 & 1.3325 .26 & \\
\hline \multirow{2}{*}{ \% Grasa Corporal Precomp } & Hidratados & 17 & 20.31 & 6.27 & 1.62 & $-5.27 \quad 60$ & \\
\hline & Deshidrat. & 47 & 11.29 & 3.70 & .54 & -6.8417 .22 & \\
\hline \multirow[b]{2}{*}{$\%$ Agua Precomp } & Hidrat & 47 & 64.91 & 3.02 & .44 & $9.32 \quad 60$ & \\
\hline & Deshidrat. & 15 & 56.53 & 3.06 & .79 & 9.2523 .35 & \\
\hline \multicolumn{8}{|c|}{ Composición Corporal por Modalidad Deportiva } \\
\hline \multirow[b]{2}{*}{ Peso Precomp } & Lucha & 38 & 72.26 & 15.74 & 2.58 & $1.21 \quad 60$ & \\
\hline & Judo & 26 & 67.60 & & 2.64 & 1.2557 .04 & .2 \\
\hline \multirow{2}{*}{ \% Grasa Corporal Precomp } & Lucha & 38 & 13.21 & 6.52 & 1.07 & $-.42 \quad 60$ & \\
\hline & Judo & 26 & 13.87 & 485 & .97 & $-.45 \quad 59.37$ & \\
\hline \multirow{2}{*}{$\%$ Agua Precomp } & Lucha & 37 & 63.30 & 5.05 & .83 & $.86 \quad 60$ & \\
\hline & Judo & 25 & 62.25 & 4.14 & .82 & .8957 .64 & \\
\hline \multicolumn{8}{|c|}{ Composición Corporal por Género } \\
\hline \multirow{2}{*}{ Pes } & $\mathrm{H}$ & 45 & 75.57 & 14.31 & 2.18 & & \\
\hline & M & 19 & 58.63 & 7.68 & 1.76 & 6.0357 .54 & \\
\hline \multirow{2}{*}{ \% Grasa Corporal Precomp } & $\mathrm{H}$ & 45 & 11.49 & 4.66 & .71 & $-4.61 \quad 60$ & \\
\hline & M & 19 & 17.96 & 5.97 & 1.37 & -4.1928 .12 & $\cdot$ \\
\hline \multirow{2}{*}{$\%$ Agua Precomp } & $\mathrm{H}$ & 43 & 64.05 & 4.38 & .66 & $3.14 \quad 60$ & \\
\hline & M & 19 & 60.24 & 4.41 & 1.01 & 3.1334 .31 & \\
\hline
\end{tabular}

\section{al género.}

Los resultados en la variable peso corporal no muestran diferencias significativa ni a nivel de hidratación de los deportistas, ni cuando hacemos referencia a deportes, pero sin embargo sin encontramos diferencias entre género siendo esta de $p=0.004$. Igualmente se puede observar en la tabla 1 como en esta etapa cercana a la propia competición, el número de atletas que se encuentran deshidratados es mayor que los que están en valores óptimos de hidratación. La media de los porcentajes de grasa de los luchadores es menor que la de los judokas, al igual que ocurre con el peso corporal.

\section{Discusión}

Las conductas alimentarias han preocupado en el ámbito deportivo en muchos casos sin llegar a mostrar la importancia real que se merecen. La calidad de la dieta, incluso en diversos momento de evolución de los deportistas, sumado a la actividad física que realizan, pasa a ser un factor fundamental independientemente de su género (Pinel et al. 2017). La cuidada selección de alimentos ricos en nutrientes es esencial cuando la ingesta energética se limita para intentar reducir el peso corporal por exigencias del propio deporte. Los atletas deberían tratar de conseguir reemplazar las reservas de carbohidratos entre sesiones de entrenamiento y acostumbrar a su cuerpo de manera paulatina para así prosperar en la propia competición.

Muchos deportistas equivocan sus pasos a la hora de decidir la restricción de alimentos en su dieta, por lo que es necesario normalizar la ingesta de micro y macronutrientes (Filaire et al. 2001; Braun, 2010).

Hemos observado como los datos nos han aportado una visión más directa de una realidad que se convierte en desórdenes alimenticios. Los deportistas de este tipo de deportes por categorías de peso, llegado el momento pre-competitivo, necesitan llegar al peso de competición a costa de estar deshidratados. Cuando hablamos de deshidratación, no sólo debemos hacer referencia a la falta de líquidos en el organismo del sujeto, sino a la falta y restricción alimenticia tan radical que se realiza por este colectivo.

La deshidratación en los deportistas de deportes de combate, ya sean judokas y/o luchadores es muy elevada cuando se acerca la etapa pre-competitiva en búsqueda del peso de competición a costa de disminuir sus niveles óptimos en la ingesta de su dieta habitual como deportistas. Dentro de estos efectos drásticos al organismo, son los hombres los que superan a las mujeres de media en las actuaciones nutricionales, aun siendo significativas las dos. Las saunas extremas con plásticos, los vómitos y diuréticos junto con la restricción alimenticia en los días previos a la competición provocan estos resultados que afectan a la propia competición y a los valores de fuerza y potencia de los deportistas (Calvo, 2013).

\section{Conclusiones}

Conocer que situaciones son las que se suceden de manera habitual en deportes como el judo o la lucha, donde por exigencias de las categorías de peso es necesario someter al organismo a constantes esfuerzos y cambios, nos lleva a entender los efectos a corto y largo plazo en el rendimiento que esto puede generar en el atleta, tanto para los entrenamientos como para la propia competición, intentando conseguir con ello la optimización de los resultados deportivos. La correcta adecuación de ingesta de nutrientes durante toda la temporada y el aprendizaje de hábitos nutricionales que permitan al deportista poder entrenar al máximo nivel durante los ciclos que conforman su planificación, debe ser el objetivo del cuerpo técnico.

Los parámetros de composición corporal se ven afectados por los malos hábitos de estos atletas a la hora de acercarse al peso de competición, en el que se combina un desconocimiento de los daños que sufre el organismo debido a prácticas de deshidratación y escasa aportación de nutrientes por parte de deportistas y entrenadores y al régimen de entrenamientos seguidos por los atletas. Estas situaciones afectan al rendimiento de los deportistas en el ámbito competitivo, y es más acuciante cuando este proceder se repite en diversos momentos de la temporada, (de dos a seis veces) con el perjuicio que ello supone. Los desórdenes alimentarios que se producen en los deportistas de deportes de combate con categorías de peso en los microciclos precompetitivos, conforman uno de los aspectos más problemáticos y de difícil solución en disciplinas deportivas como el judo o la lucha, por lo que es fundamental trabajar en esos malos hábitos

Igualmente este desencadenante de problemas se suma a otros de nivel fisiológico y psicológico que pueden hacer que las expectativas deportivas se vean truncadas por una mala preparación nutricional. La tensión, la ira, la falta de concentración o la confusión del deportista en 
las acciones de competición, sumada a la fatiga y a la reducción de la fuerza muscular son significativamente elevadas como consecuencia de la restricción alimenticia por causas de la pérdida de peso corporal.

El contexto deportivo, junto con las características del propio deporte, son factores determinantes de observación y análisis. Sumado a ellos, nos encontramos el momento de la temporada donde la presión aumenta en el deportista, siempre que por las circunstancias, se vea obligado a practicar algún tipo de dieta, y es ahí donde la figura del entrenador o equipo técnico como observador y controlador de situaciones negativas que afecten al atleta es fundamental, (Díaz, 2005) para evitar los trastornos en las conductas alimenticias que tanto afectan a este tipo de deportistas.

\section{Referencias}

Boisseau, N; Vera-Pérez, S; Poortmans, J (2005) Food and fluid intakes in female adolescent judo athletes before competition. Pediatric Exercise Science. Vol. 17, 1:62-71

Braun, H. (2010) Sports nutritional characteristics classified by weight category. Aktuelle Ernahrungsmedizin. Vol. 35(4): 178-182

Calvo, B (2013)Análisis de los indicadores cinemáticos en deportistas de deportes de combate en situación de deshidratación precompetitiva. Tesis Doctoral. Facultad de Ciencias del Deporte de Toledo. Universidad de Castilla-La Mancha

Clemente Suárez, V; González Ravé, J.M. (2010) Modificaciones en la composición corporal después de realizar una prueba de ultrarresistencia de 1700 km en bicicleta de montaña. Revistas Ciencias del Deporte 14, año 6, Vol 5: 95-99. Murcia

De Barros, J; Fernandes, A.P; Oliveira, J.V.S; Stulbach, T.E; García, L.D; Peron, A.N, Dattilo, M. (2010) Evaluation of water loss in judo training and its relationship with subjective hunger and appetite scores. Revista Brasileira De Medicina Do Esporte. Vol. 16 (6): 408-412

Díaz Ceballos, I (2005) Propuesta de un programa de prevención de trastornos de la conducta alimentaria para entrenadores. Cuadernos de Psicología del Deporte, Vol 5, 1 y 2

Dossil, J (2004) Psicología de la actividad física y el deporte. Editorial McGraw-Hill. Madrid

Engels, S.G, Johnson, C; Power, P.S; Crosby, R.D; Wonderlich, S.A Wittrock, D.A; Mitchell, J.E (2003) Predictors of disordered eating in a simple of elite Division college athletes. Eating Behaviors. Vol. 4 (4): 333-343

Filaire, E; Maso, F; Degoutte, F; Jouanel, P; Lac, G. (2001) Food Restriction, performance, Psychological state and lipid values in judo athletes. International Journal Sport Medicine. Vol. 22: 454459

Franseen, L.M. (1997) Environmental preassures, personality factors and their relationship to eating disorders in elite female athletes. Dissertation Abstracts International Section B: The Sciencie and Engineering. Vol. 57 (7-B): 4781

Juzwiak, C.R; Ancona López, F. (2004) Evaluation of knowledge about nutrition and dietary recommendations of the Brazilian adolescent athletes coaches. International Journal of Sport Nutritional Exercise Metab. Vol. 14(2): 222-235

Kiningham, R; Gorenflo, D. (2001) Weight loss methods of high school wrestlers. Medicine Science of Sports Exercises. Vol. 33 (5): 810813

Márquez, S (2008) Trastornos alimentarios en el deporte: factores de riesgo, consecuencias sobre la salud, tratamiento y prevención. Nutrición Hospitalaria Vol. 23 (3): 183-190

Marrodán, M; Santos, M; Mesa, M; Cabañas, M; González-Montero, M; Pacheco,J.L. (2007) Técnicas analíticas en el estudio de la composición corporal.Antropometría frente a sistemas de bioimpedancia bipolar y tetrapolar. Nutrición Clínica y Dietética Hospitalaria Vol. 27 (3)

Martín-Moreno, V; Gómez, B; Antoranz, M.B; Fernández, S; Gómez de la Cámara, A; De Oya, M. (2001) Validacaión del monitor de medición de la grasa corporal por impedancia bioeléctrica OMRON BF 300. Revista de Atención Primaria. Vol. 8 (3):

Matías, C.N; Santos, D.A; Monteiro, C.P; Silva,A.M; Raposa, M.D.F; Martins, F; Sardinha, L.B; Bicho, M; Laires, M.J(2010) Magnesium and streght in elite judo athletes according to intracelular wáter changes. Magnesium Research. Vol. 23 (3): 138-141

Müller, W. (2009) Towards research-based approaches for solving body composition problems in sport: Ski jumping as a heuristic example. British Journal of Sport Medicine. Vol. 43 (13): 10131019

Ööpik, V; Timpmann, S; Burke, A; Hannus, I. (2013) Hydration status of Greco-Roman wrestlers in authentic pre-competition situation. Applied Physiology, Nutrition, and Metabolism Vol. 10

Pinel Martínez, C; Chacón Cuberos, R; Castro Sánchez, M; Espejo Garcés, T; Zurita Ortega, F; Pérez Cortés, A. (2017)Diferencias de género en relación con el índice de masa corporal, calidad de la dieta y actividades sedentarias en niños de 10 a 12 años. Revista Retos 31, 176-180

Rodríguez Martín, A; Martínez Nieto, J.M; Novalbos Ruíz, J.P; Ruíz Jiménez, M.A; Jiménez Benítez, D (1999) Ejercicio Físico y Hábitos alimentarios: un estudio en adolescentes de Cádiz. Revista Esp. Salud Pública Vol. 73: 81-87

Rodríguez, N.R; DiMarco, N.M (2009) Nutrition and athletic performance: vitamins and minerals. Medscape

Sundgot-Borgen, J; Torstveit, M. (2004) Prevalence of eating disorders in elite atlethes is higher than in the general population. Clinical Journal of Sport Medicine. Vol. 14 (1): 25-32

Thield, A; Gottfried, H; Hesse, F.W (1993) Subclinical eating disorder in male athletes: a study of the low weight category in rowers and wrestlers. Acta Psychiatrica Scandinavica, 88 (4): 259-265

Úbeda, N; Palacios Gil-Antuaño, N; Montalvo Zenarruzabeitia, Z; García Juan, B; García, A; Iglesias Gutierrez, E; (2010) Hábitos alimenticios y composición corporal de deportistas de élite pertenecientes a disciplinas de combate. Nutrición Hospitalaria Vol. 25, $\mathrm{N}^{\circ} 3$

\section{(Footnotes)}

IAnorexia Nerviosa: trastorno de la conducta alimentaria provocado por el propio enfermo que provoca su propia disminución de peso corporal disminuyen voluntariamente total o parcialmente el consumo de alimentos y seleccionan aquellos que ayuden a la reducción de su peso corporal.

${ }^{2}$ Bulimia Nerviosa: trastorno alimentario y psicológico durante el cual, una persona modifica su alimentación consumiendo un exceso de comida en periodos de tiempo muy cortos para después buscar eliminar el exceso de alimento a través de ayunos, vómitos o laxantes.

${ }^{3}$ Anorexia Atlética: trastorno provocado por el deportista para mejorar su figura reduciendo su peso corporal de manera voluntaria a través de la disminución de los alimentos, sumado a largos periodos de actividad deportiva.

${ }^{4}$ Vigorexia: también conocido como dismorfia muscular, trastorno alimentario caracterizado por la obsesión por el físico, a veces referido como «anorexia nerviosa inversa» 0 «complejo de adonis».

${ }^{5}$ Kumikata: agarre en judo. El agarre normal se realiza con una mano a la manga y la otra a la solapa del contrario. En competición los agarres varían en función de las técnicas especiales (tokui-waza) de los competidores.

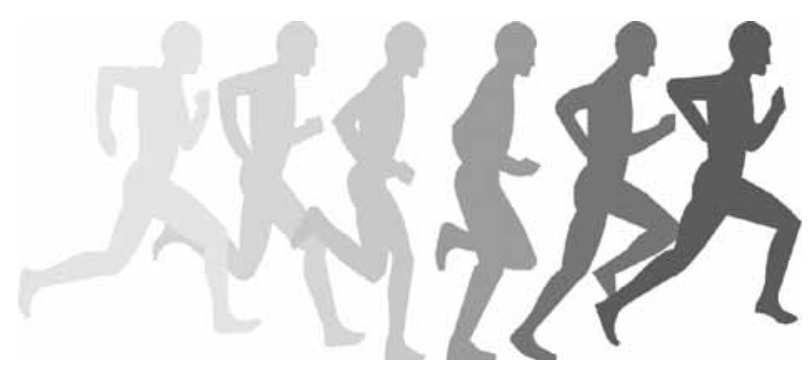

